\title{
Follicular thyroid carcinoma with NRAS Q61K and GNAS R201H mutations that had a good ${ }^{131}$ I treatment response
}

\author{
Jin-Ying Lu', Po-Ju Hung', Pei-Lung Chen ${ }^{1,2,3,4,5}$, Ruoh-Fang Yen ${ }^{6}$, Kuan-Ting Kuo ${ }^{7,8}$, \\ Tsung-Lin Yang $^{9}$, Chih-Yuan Wang ${ }^{1}$, Tien-Chun Chang ${ }^{1,10}$, Tien-Shang Huang ${ }^{1,10,11,12}$ \\ and Ching-Chung Chang 1,10,13,14 \\ 'Division of Endocrinology and Metabolism, Department of Internal Medicine, \\ ${ }^{2}$ Department of Medical Genetics, National Taiwan University Hospital, Taipei 100, Taiwan, \\ ${ }^{3}$ Graduate Institute of Medical Genomics and Proteomics, ${ }^{4}$ Graduate Institute of Clinical Medicine, ${ }^{5}$ Research Center \\ for Developmental Biology and Regenerative Medicine, ${ }^{6}$ Department of Nuclear Medicine, ${ }^{7}$ Graduate Institute of \\ Pathology, College of Medicine, National Taiwan University, Taipei 100, Taiwan, \\ Departments of ${ }^{8}$ Pathology, ${ }^{9}$ Otolaryngology, National Taiwan University Hospital, Taipei 100, Taiwan, \\ Departments of ${ }^{10}$ Medicine, ${ }^{11}$ Social Medicine, College of Medicine, National Taiwan University, Taipei 100, Taiwan, \\ ${ }^{12}$ Department of Medicine, Cathay General Hospital, Taipei 106, Taiwan, \\ ${ }^{13}$ Department of Internal Medicine, China Medical University Hospital, Taichung 404, Taiwan and \\ ${ }^{14}$ Department of Internal Medicine, China Medical University, Taichung 404, Taiwan
}

\author{
Correspondence \\ should be addressed \\ to J-Y Lu or C-C Chang \\ Emails \\ jylu@ntu.edu.tw or \\ changchingchung@ \\ gmail.com
}

\section{Summary}

We report a case of follicular thyroid carcinoma with concomitant NRAS p.Q61K and GNAS p.R201H mutations, which manifested as a $13.5 \mathrm{~cm}$ thyroid mass with lung, humerus and T9 spine metastases, and exhibited good response to radioactive iodine treatment.

\section{Learning points:}

- GNAS p.R201H somatic mutation is an activating or gain-of-function mutation resulting in constitutively activated Gs-alpha protein and downstream CAMP cascade, independent of TSH signaling, causing autonomously functioning thyroid nodules.

- NRAS p.Q61K mutations with GNAS p.R201H mutations are known for a good radioactive iodine treatment response.

- Further exploration of the GNAS-activating pathway may provide therapeutic insights into the treatment of metastatic follicular carcinoma.

\section{Background}

We report a 79-year-old man with follicular thyroid carcinoma arising from a history of Graves' disease. Two mutations, NRAS p.Q61K and GNAS p.R201H, were detected by the Ion Torrent Personal Genome Machine (PGM) covering > 400 amplicons in 46 genes, and were confirmed by direct Sanger sequencing. The patient's thyroid cancer manifested as a $13.5 \mathrm{~cm}$ thyroid mass with minimal lung, humerus and $\mathrm{T} 9$ spine metastases, and exhibited a very good response to the radioactive iodine treatment. We simultaneously screened another 23 fatal cases due to thyroid cancers using the same method, and found none of them carrying the GNAS p.R201H mutation. However, NRAS p.Q61K or NRAS p.Q61R mutations were identified in two and six of these 23 fatal cases respectively. 
We speculate that the concomitant somatic GNAS p.R201H activating mutation, in association with the relatively poor prognostic NRAS p.Q61K mutation of follicular thyroid carcinoma in our patient, possibly contributed to the relatively low metastatic burden, the increased radioactive iodine uptake and the good response to radioactive iodine treatment in our patient. We suggest that further exploration of the pathways involving the Gs $\alpha$ activation and related approaches leading to higher radioactive iodine treatment efficacy may provide significant insights into the pathogenesis and treatment of differentiated thyroid cancers.

\section{Case presentation}

This 79-year-old man had a history of Graves' disease for 5 years. The free thyroxine $\left(\mathrm{FT}_{4}\right)$ was $2.81 \mathrm{ng} / \mathrm{dl}$ (0.89-1.75), the thyroid-stimulating hormone (TSH) was $<0.004 \mu \mathrm{IU} / \mathrm{ml}(0.4-4.0)$, and the TSH-receptor antibody was $39.7 \%(<10 \%)$ on June 22,2007 at the initial visit. He had received endocrinological outpatient clinical treatment and had been followed up regularly for 5 years since then. Left nodular goiter was noted on physical examination, and thyroid ultrasonography on April 4, 2009 revealed a bilateral multi-nodular goiter, and a large $(6.1 \times 5.47 \times 4.53 \mathrm{~cm})$ left thyroid mass with trachea deviation to the right. Fine needle aspiration (FNA) on April 15, 2009 and August 12, 2009 were non-diagnostic. Thyroid ultrasonography on March 2, 2011 showed a left thyroid mass $(6.09 \times 5.67 \times$ $2.64 \mathrm{~cm}$ ) with trachea deviation to the right and left thoracic extension (Fig. 1A). Repeated FNA cytology on August 16, 2011 revealed follicular cells with colloid.

However, he noted that his voice became hoarse on November, 2011.

\section{Investigation}

He was referred to an ENT specialist on November 25, 2011, and endoscopic examination of the larynx showed left vocal cord palsy. Head and neck ultrasonography on December 23, 2011 revealed a huge thyroid mass on the left side with trachea deviation to the right, and at least three lymph nodes at the left neck level II, measuring $1.1 \times 1.1 \mathrm{~cm}, \quad 1.4 \times 0.7 \mathrm{~cm}$, and $0.8 \times 0.56 \mathrm{~cm}$ were demonstrable. FNA again showed clusters of follicular cells and colloid. MRI on December 14, 2011 revealed a huge mass probably arising in the left thyroid with compression and displacement of the trachea and adjacent structures measuring about $13.6 \mathrm{~cm}$ at the longest dimension (Fig. 1B).

\section{Treatment}

A wide excision of the left thyroid tumor and intrathoracic goiter, and dissection of the left supraomohyoid neck lymph node was performed on January 4, 2012. However, after extubation, chylous leak was noted and wound direct compression was applied, and so he was treated with elemental diet and intravenous infusion of albumin. Subcutaneous air leak at the previous tumor site was noted, and subcutaneous catheter was implanted to remove the air. There was no further air leak 5 days after operation. The drainage amount decreased gradually and the drain tube was removed 16 days later. The pathology revealed thyroid follicular neoplasm favoring malignancy, without neck lymph node metastasis (Fig. 1C and D).

Staging work-ups including lung and abdominal computed tomography (CT) without contrast was performed on January 18, 2012. CT scan showed an indeterminate tiny nodule at the lingular segment of the left upper lung focal osteolytic lesions with coarse trabeculation at T7 and T9 vertebral bodies and small hypodense lesions at both lobes of the liver, nature indeterminate, suspected hepatic cysts. Bone scan on January 19, 2012 showed no definite osteoblastic bone metastasis.

He underwent total thyroidectomy and bilateral neck dissection on February 6, 2012, and the pathology reported no malignancy in the right thyroid gland and the dissected lymph nodes. Thirty $\mathrm{mCi}$ of ${ }^{131} \mathrm{I}$ was given on March 9, 2012, and the whole body scan showed residual viable malignancy or residual functioning thyroid tissue at anterior neck, and metastatic lesions at $\mathrm{T} 9$ spine and bilateral lower lungs (Fig. 2A and B). However, he had no back pain or respiratory symptoms.

As part of the treatment for metastatic thyroid follicular carcinoma, he received $200 \mathrm{mCi}$ of ${ }^{131} \mathrm{I}$ treatment. Thyroglobulin levels decreased from $>300 \mathrm{ng} / \mathrm{ml}$ (the TSH was $22 \mu \mathrm{IU} / \mathrm{ml}$ after discontinuing $\mathrm{L}_{-} \mathrm{T}_{4}$ for 4 weeks) before the $30 \mathrm{mCi}$ of ${ }^{131}$ I treatment, to $35 \mathrm{ng} / \mathrm{ml}$ (the TSH was $>75 \mu \mathrm{IU} / \mathrm{ml}$ after two $1 \mathrm{mg}$ thyrogen i.m. administrations, and the thyroglobulin antibody was $1.22 \mathrm{IU} / \mathrm{ml}(<14.4)$ ) on May 3, 2012 (Fig. 2C). The whole body scan showed multiple metastases at the right humerus, T9 spine and bilateral lungs.

\section{Outcome and follow-up}

Follow-up of ${ }^{131}$ I whole body scan on May 22, 2013 showed probable treatment effects with residual ${ }^{131} \mathrm{I}$-avid malignancy at T9 spine; the simultaneous stimulated TSH 
A
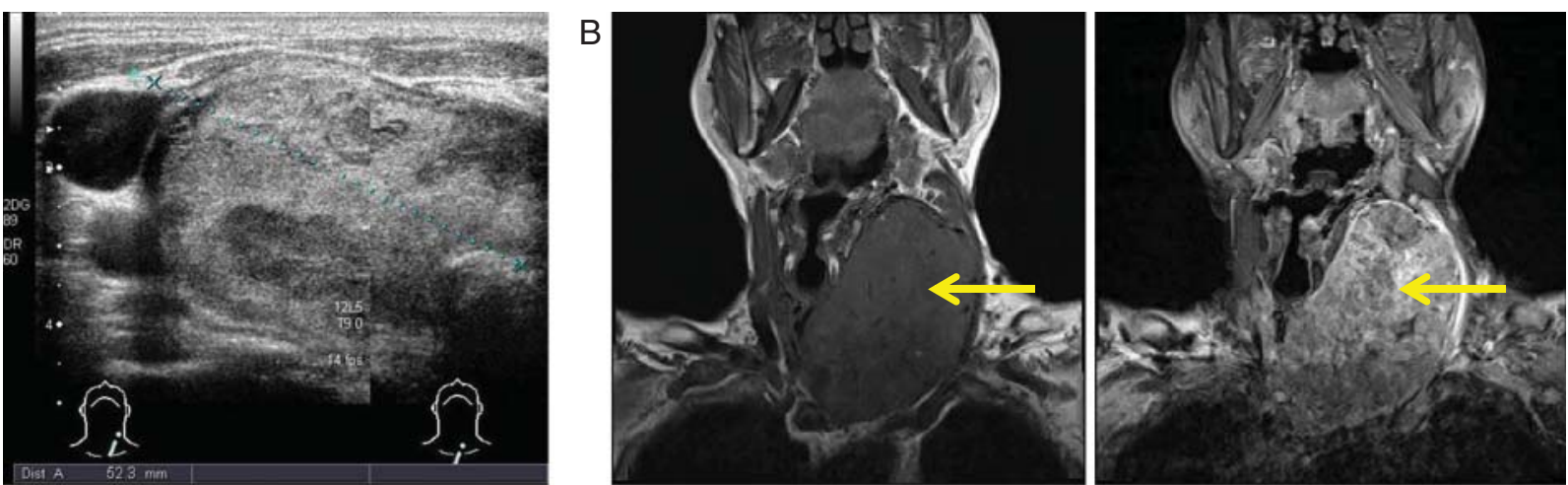

C
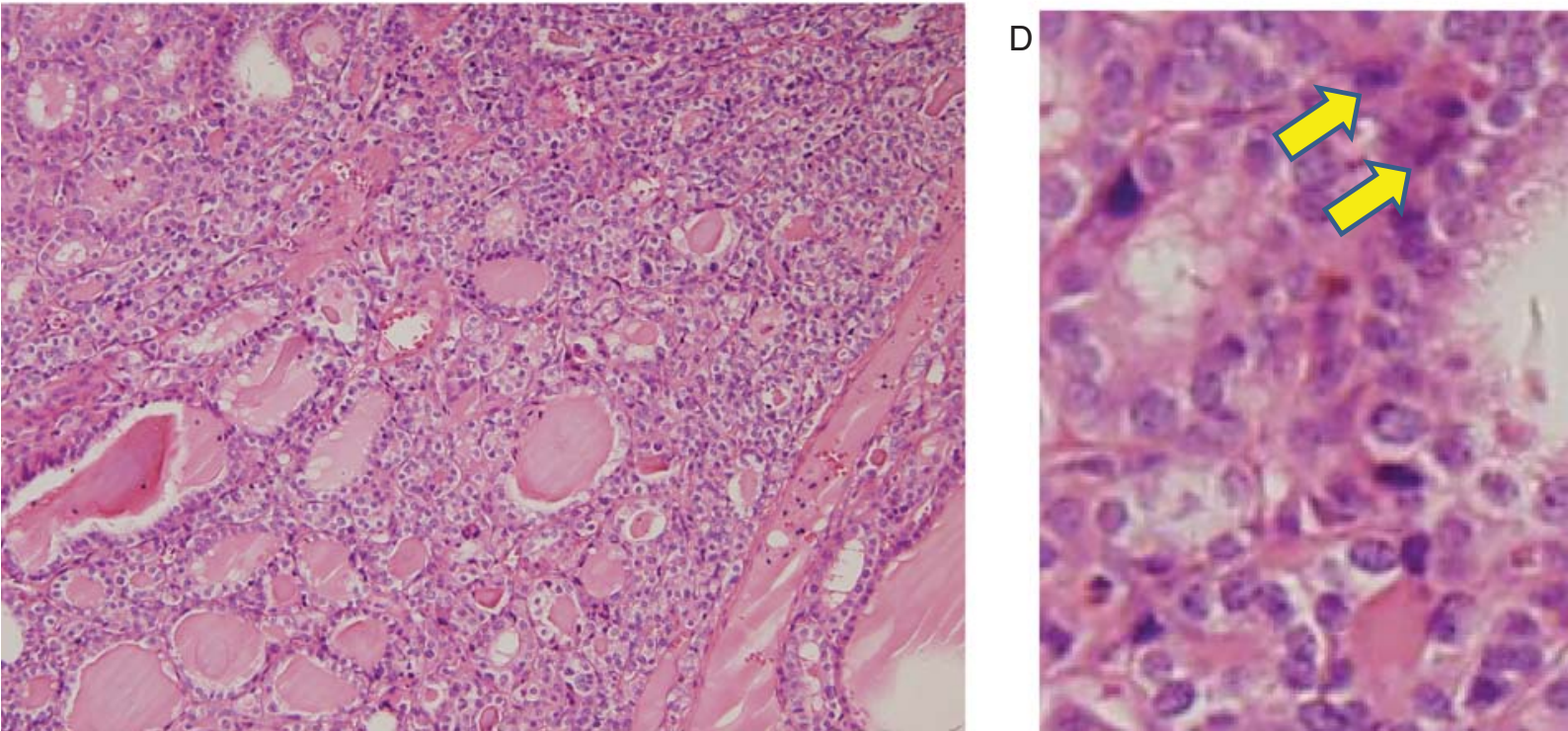

\section{Figure 1}

(A) Thyroid ultrasonography on July 18, 2007 showed a huge left thyroid mass with trachea deviation to the right. (B) Head and neck MRI, coronal view, T1-weighted image without contrast (left), and flow compensation/fat saturation image with contrast (right), on December 13, 2011 showed a huge mass arising in the left thyroid with compression, displacement of the trachea and adjacent structures (yellow arrows). It measures about $13.6 \mathrm{~cm}$ in the longest dimension. (C) Microscopic examination showed that the tumor is composed of heterogeneous architecture and patterns range from goiter-like follicular glands,

level after two doses of i.m. injection of $0.9 \mathrm{mg}$ of thyrogen for two consecutive days was $21.5 \mu \mathrm{IU} / \mathrm{ml}$, and the thyroglobulin declined to $1.83 \mathrm{ng} / \mathrm{ml}$. The last follow-up on March 21, 2015 revealed that the TSH level was $1.84 \mu \mathrm{IU} / \mathrm{ml}$, and the thyroglobulin was $0.966 \mathrm{ng} / \mathrm{ml}$. He remained symptom free during outpatient clinic follow-ups.

We extracted genomic DNA of both the left thyroid tumor and the right side normal thyroid tissues, from the formalin-fixed, paraffin embedded (FFPE) surgical tissues sent for pathology examinations using WaxFree (TrimGen) DNA extraction kit as described previously (1), after micro-follicles and solid, trabecular, insular growth. The follicular glands contain eosinophilic colloid and arrange from compact micro-follicles to markedly distended glands. (D) In the areas with solid growth, marked nuclear hyperchromasia, and pleomorphism are noted. There are tumor protrusions into sinusoid spaces of the capsule, but no thick-walled vascular invasion can be demonstrated. Mitotic activity counts up to three per ten high power fields (yellow arrows with blue margin). The diagnosis of a follicular carcinoma is favored.

obtaining informed consent from the patient. The DNA quality was verified by agarose gel electrophoresis and Nanodrop spectrophotometer at 260/280 nm. Genetic analysis of the extracted genomic DNA using Ion Torrent PGM covering $>400$ amplicons in 46 genes was carried out as reported (2). Results were available for analysis after sequencing on the 316 chip using Ion Torrent PGM System and Ion PGM Sequencing 200 Kit. Data analysis, including alignment to the hg19 human reference genome and variant calling, was conducted using Torrent Suite Software v3.0 and then viewed through a standard web browser. The analysis revealed two specific mutations 


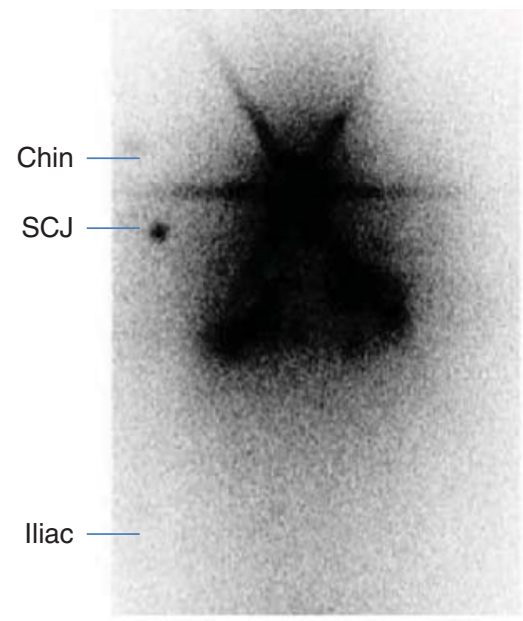

C

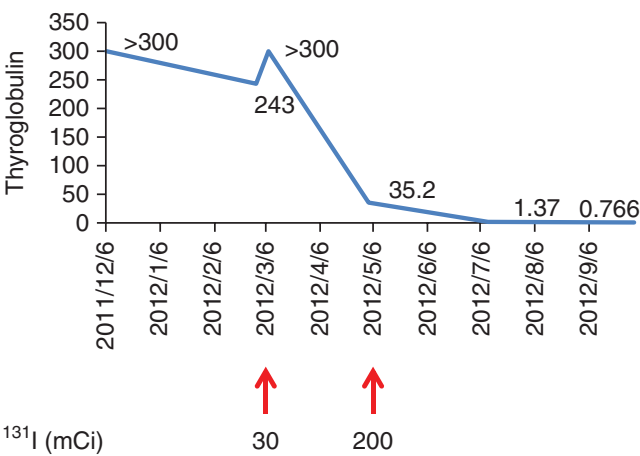

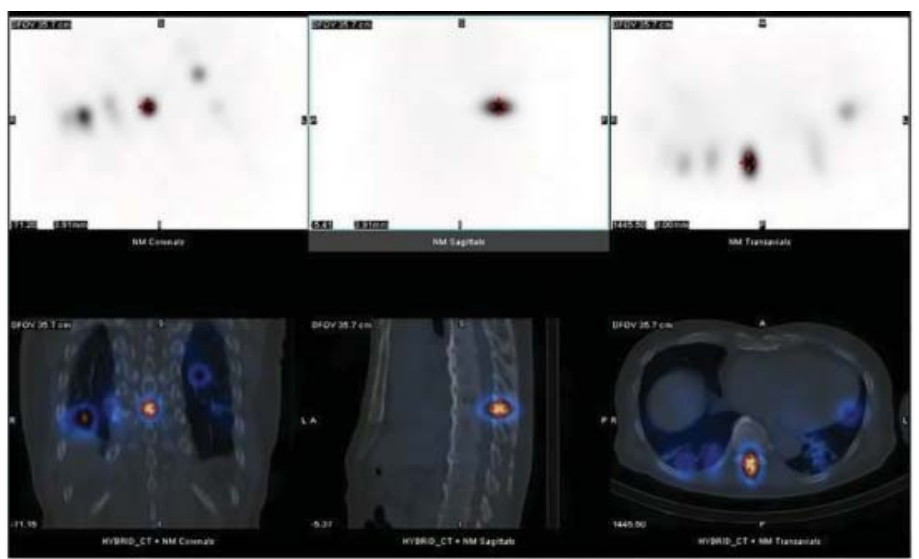

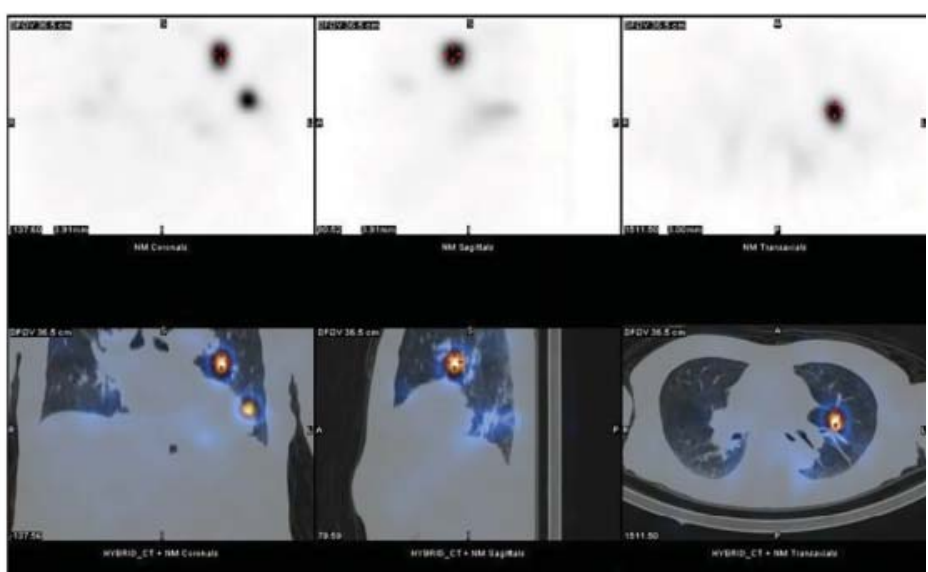

Figure 2

The whole body radionuclide scintigraphy $(\mathrm{A})$ and regional single-photon emission computed tomography (SPECT) (B) were performed with high energy gamma camera at 8 days after treatment. A focal hot area at

in the tumor tissue, the NRAS c.181C $>$ A (p.Q61K) and GNAS c.602G > A (p.R201H) mutations, but not in the non-tumor counterpart. The target sequences in these two genes were amplified, and the PCR products of genomic DNA were analyzed on a DNA Analyzer and the sequence reactions were carried out using ABI BigDye Terminator chemistry on a Thermal Cycler device (ABI 3730 capillary sequencer, Applied Biosystems). The two specific mutations were thus confirmed by direct sequencing of the PCR products (Fig. 3A and B).

\section{Discussion}

Here we report a case of a huge follicular thyroid carcinoma in a patient with Graves' disease. In our patient, FNA cytology failed to reveal the presence of thyroid cancer before surgery. FNA cytology from thyroid nodules arising due to Graves' disease can pose diagnostic difficulties (3); anterior neck, a focal hot spot at spinous process of T9 spine and multiple hot spots at bilateral lower lungs were demonstrated. (C) Gradual decline of thyroglobulin levels after radioactive iodine $\left({ }^{131} \mathrm{I}\right)$ treatment.

on the other hand, based on our knowledge and previous literature reports, increased thyroid nodule size does not increase the malignancy rate for follicular neoplasms (4). The above reasons might have led to a delayed diagnosis of thyroid follicular carcinoma in our patient.

Although, the patient presented with a $13.6 \mathrm{~cm}$ large thyroid follicular carcinoma, and was immediately diagnosed to have lung and bone metastases by radioactive iodine whole body scan following total thyroidectomy, the disease course was indolent, and the biochemical response, or the thyroglobulin levels, to radioactive iodine treatment was good. Genetic mutation screens using nextgeneration sequencing, the Ion Torrent PGM, identified two somatic mutations exclusively present in the tumor tissue, the NRAS Q61K and GNAS R201H mutations. The results were further confirmed by direct Sanger sequencing. The same screens applied to 23 patients who died of thyroid cancers in our institute did not show GNAS 
A

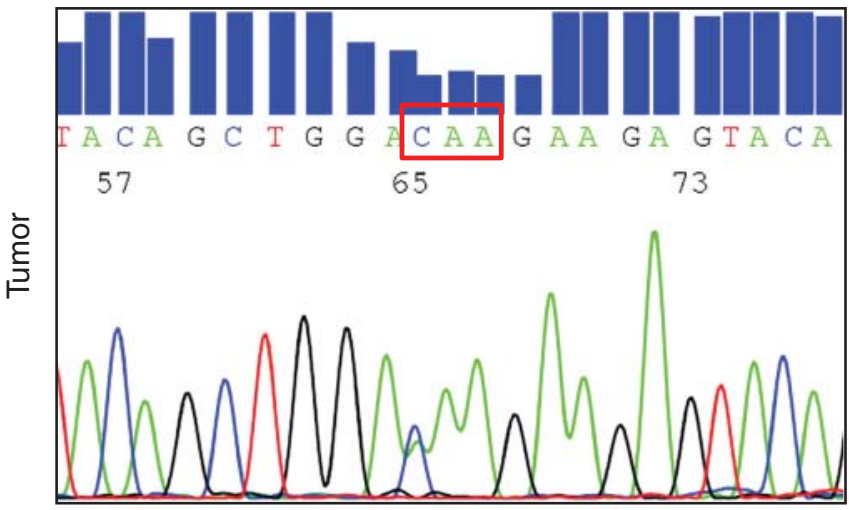

B

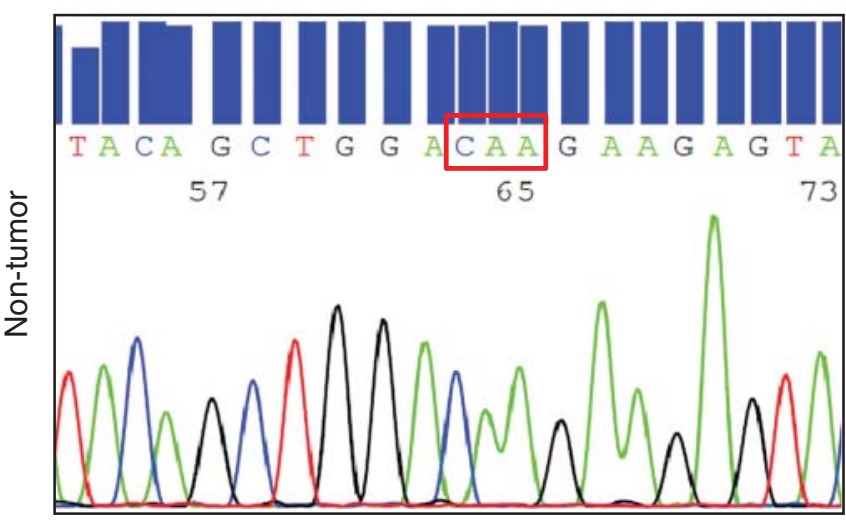

Codon c.602CGT>CAT (p.R201H)

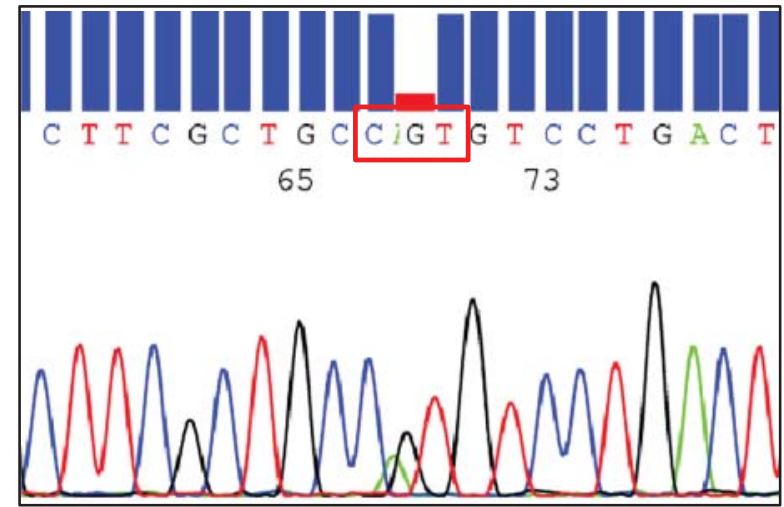

Codon c.602G (p.R201)

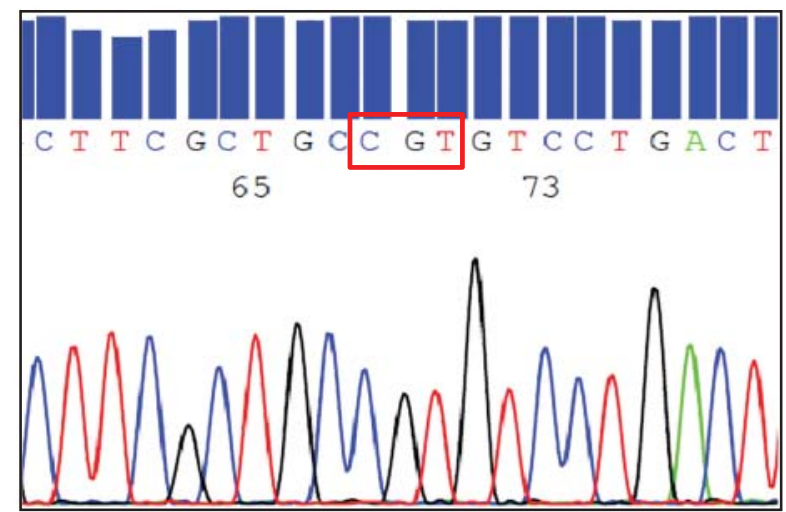

Figure 3

Four-color chromatogram showed the results of direct sequencing run of the PCR products targeting the specific DNA sequences of the tumor (A) and non-tumor $(B)$ parts of this patient's thyroid glands. The tumor part in

p.R201H mutations; however, NRAS p.Q61K or NRAS p.Q61R mutations were identified in two and six out of the 23 mortality cases respectively (J-Y Lu, P-J Hung, P-L Chen, R-F Yen, K-T Kuo, T-L Yang, C-Y Wang, T-C Chang, T-S Huang, C-C Chang, unpublished data).

Follicular thyroid carcinomas frequently harbor $R A S$ mutations, and the mutations are predominantly found in the NRAS codon 61 (3), followed by HRAS codon 61 and KRAS codon 12/13 (4). Besides, the presence of an HRAS 61 or NRAS 61 mutation has a high positive predictive value (PPV) for thyroid carcinoma diagnosis (95.5 and 86.8\% respectively), while the PPV of KRAS 12/14 mutation was associated with a significantly lower rate of carcinoma diagnosis (41.7\%) (4). It has also been reported that the NRAS codon 61 mutation in follicular carcinomas is positively associated with distant metastases and poor overall patient survival (5).

Interestingly, the thyroid follicular carcinoma in our patient harbors the second somatic mutation, the this patient, which is the left thyroid gland, harbors both NRAS p.Q61K and GNAS p.R201H mutations; while the non-tumor counterpart, or the right thyroid gland, shows WT sequences.

GNAS R201H activating mutation. It is noteworthy that pseudohypoparathyroidism is caused by germ-line inactivating mutations in GNAS scattering throughout the 13 exons that encode Gs $\alpha$ protein, and consists of $\sim 45 \%$ frameshift, 28\% missense, 14\% nonsense, and 9\% splicesite mutations, $\sim 3 \%$ in-frame deletions or insertions, and $\sim 1 \%$ whole or partial gene deletions. These mutations lead to constitutively decreased cAMP signaling and multiple endocrine dysfunctions, including morphological and functional thyroid involvement (6). On the contrary, the GNAS p.R201H somatic mutation in our patient, similar to those that occurred in McCune-Albright syndrome (MAS) (7), is an activating or gainof-function mutation resulting in constitutively activated Gs $\alpha$ protein and downstream cAMP cascade, independent of TSH signaling, causing autonomously functioning thyroid nodules in both iodine-deficient (8) and iodinerich regions (9). Though being a rare occurrence in thyroid cancer (10), GNAS p.R201H mutation might have played 
an important role in regulating tumor progression and radioactive iodine uptake in this patient.

Notably, in our patient TSH levels did not rise higher than $30 \mu \mathrm{IU} / \mathrm{ml}$ (stimulated TSH was $22 \mu \mathrm{IU} / \mathrm{ml}$ ) after L- $\mathrm{T}_{4}$ withdrawal for 4 weeks was noted, which possibly is related to the autonomously functioning metastatic thyroid carcinomas. We recommend using recombinant human TSH (rhTSH; thyrogen) for boosting the TSH level, instead of thyroid hormone withdrawal, before radioactive iodine treatment in this situation.

We conclude that the concomitant somatic GNAS p.R201H activating mutation, in association with the relatively poor prognostic NRAS p.Q61K mutation of follicular thyroid carcinoma in our patient, possibly contributed to the increased radioactive iodine uptake and the good response to radioactive iodine treatment in our patient. We suggest that further exploration of the pathways involving Gs $\alpha$ activation and related approaches leading to higher radioactive iodine treatment efficacy may provide significant insights into treatment of thyroid cancers.

\section{Declaration of interest}

The authors declare that there is no conflict of interest that could be perceived as prejudicing the impartiality of the research reported.

\section{Funding}

This study was supported by National Taiwan University Hospital research grants NTUH. 101-001860 and NTUH. 102-002146 to J-Y Lu.

\section{Patient consent}

Written informed consent has been obtained from the patient for publication of the case report and accompanying images.

\section{Author contribution statement}

J-Y Lu and T-L Yang are the named physicians of the patient who provided medical care and follow-ups of the patient at the outpatient clinics. T-L Yang performed total thyroidectomy and lymph node dissection for this patient, K-T Kuo viewed the pathology and made diagnosis for the patient. J-Y Lu, R-F Yen and P-J Hung took care of the patient during the admission for radioactive iodine treatment. J-Y Lu, P-L Chen, C-Y Wang, T-C Chang, T-S Huang, C-C Chang, and K-T Kuo helped with designing the study, discussing the genetic findings and writing the paper. The Laboratory of Cancer Genomic Medicine of LIHPAO Life Science CO., Ltd. has assisted with the next-generation sequencing and Sanger's sequencing for the patient.

\section{Acknowledgements}

We dedicate this paper to the memory of our dear colleague, Yu-Yi Lin. The author expresses gratitude to the Laboratory of Cancer Genomic Medicine of LIHPAO Life Science CO., Ltd. for their kindness in assisting with the next-generation sequencing and Sanger's sequencing. The completion of this undertaking acknowledges their participation and professionalism in the analysis of the data.

\section{References}

1 Turashvili G, Yang W, McKinney S, Kalloger S, Gale N, Ng Y, Chow K, Bell L, Lorette J, Carrier M et al 2012 Nucleic acid quantity and quality from paraffin blocks: defining optimal fixation, processing and DNA/RNA extraction techniques. Experimental and Molecular Pathology 92 33-43. (doi:10.1016/j.yexmp.2011.09.013)

2 Singh RR, Patel KP, Routbort MJ, Reddy NG, Barkoh BA, Handal B, Kanagal-Shamanna R, Greaves WO, Medeiros LJ, Aldape KD et al 2013 Clinical validation of a next-generation sequencing screen for mutational hotspots in 46 cancer-related genes. Journal of Molecular Diagnostics 15 607-622. (doi:10.1016/j.jmoldx.2013. 05.003)

3 Hwang TS, Kim WY, Han HS, Lim SD, Kim WS, Yoo YB, Park KS, Oh SY, Kim SK \& Yang JH 2015 Preoperative RAS mutational analysis is of great value in predicting follicular variant of papillary thyroid carcinoma. BioMed Research International 2015 697068. (doi:10.1155/2015/697068)

4 Radkay LA, Chiosea SI, Seethala RR, Hodak SP, LeBeau SO, Yip L, McCoy KL, Carty SE, Schoedel KE, Nikiforova MN et al 2014 Thyroid nodules with KRAS mutations are different from nodules with NRAS and HRAS mutations with regard to cytopathologic and histopathologic outcome characteristics. Cancer Cytopathology 122 873-882. (doi:10.1002/cncy.21474)

5 Jang EK, Song DE, Sim SY, Kwon H, Choi YM, Jeon MJ, Han JM, Kim WG, Kim TY, Shong YK et al 2014 NRAS codon 61 mutation is associated with distant metastasis in patients with follicular thyroid carcinoma. Thyroid 24 1275-1281. (doi:10.1089/thy.2014.0053)

6 Turan S \& Bastepe M 2015 GNAS Spectrum of Disorders. Current Osteoporosis Reports 13 146-158. (doi:10.1007/s11914-015-0268-x)

7 Boyce AM \& Collins MT. Fibrous dysplasia/McCune-Albright syndrome. In GeneReviews(R) Eds RA Pagon, MP Adam, HH Ardinger, SE Wallace, A Amemiya, LJH Bean, TD Bird, CR Dolan, CT Fong, RJH Smith, K Stephens. Seattle (WA), 1993.

8 Georgopoulos NA, Sykiotis GP, Sgourou A, Papachatzopoulou A, Markou KB, Kyriazopoulou V, Papavassiliou AG \& Vagenakis AG 2003 Autonomously functioning thyroid nodules in a former iodinedeficient area commonly harbor gain-of-function mutations in the thyrotropin signaling pathway. European Journal of Endocrinology/ European Federation of Endocrine Societies 149 287-292. (doi:10.1530/ eje.0.1490287)

9 Nishihara E, Amino N, Maekawa K, Yoshida H, Ito M, Kubota S, Fukata S $\&$ Miyauchi A 2009 Prevalence of TSH receptor and Gsalpha mutations in 45 autonomously functioning thyroid nodules in Japan. Endocrine Journal 56 791-798. (doi:10.1507/endocrj.K09E-073)

10 Nikiforova MN, Wald AI, Roy S, Durso MB \& Nikiforov YE 2013 Targeted next-generation sequencing panel (ThyroSeq) for detection of mutations in thyroid cancer. Journal of Clinical Endocrinology and Metabolism 98 E1852-E1860. (doi:10.1210/jc.2013-2292)
Received in final form 11 December 2015 Accepted 5 January 2016 